\title{
Takotsubo cardiomyopathy mimicking acute ST-segment elevation myocardial infarction in a 28-year-old woman
}

Laxman Dubey, ${ }^{*}$ Sanjib Kumar Sharma, Samir Gautam, Sachin Dhungel, Shankar Laudari

Department of Cardiology, College of Medical Sciences and Teaching Hospital, Bharatpur, Nepal

\author{
Accepted on \\ December 29th, 2013 \\ DOI Name \\ http://dx.doi.org/10.3126/jaim.v3i1.10703

\section{Keywords} \\ acute myocardial infarction; chest pain: \\ intrauterine fetal death; Takotsubo \\ cardiomyopathy \\ Citation \\ Laxman Dubey, Sanjib Kumar Sharma, Samir \\ Gautam, Sachin Dhungel, Shankar Laudari. \\ Takotsubo cardiomyopathy mimicking acute ST- \\ segment elevation myocardial infarction in a \\ 28-year-old woman. Journal of Advances in Internal \\ Medicine 2014;03(01):38-41.
}

\begin{abstract}
Takotsubo cardiomyopathy is a rare type of nonischemic cardiomyopathy accompanied by a transient ballooning and akinesis of the apical segment of left ventricle. Here, we report a 28-year-old woman who presented with acute chest pain after an emotional stress. Electrocardiography showed STsegment elevation in leads II, III, aVF, V3-V6. Echocardiography, coronary angiography and left ventriculography showed typical features of Takotsubo cardiomyopathy.
\end{abstract}

\section{INTRODUCTION}

Takotsubo cardiomyopathy (TCM) is a rare type of nonischemic cardiomyopathy accompanied by a transient ballooning and akinesis of the apical segment of left ventricle (LV). It is also known as transient LV apical ballooning syndrome, stressinduced cardiomyopathy, or broken heart syndrome. TCM was first described as a clinical entity in Japan in the early 1990's by Dote and colleagues. ${ }^{1}$ TCM was named after the original Japanese octopus trap which is a fishing jar with a narrow neck and wide base used to trap octopus. In Japanese, tako means octopus and tsubo means pot, hence the name. ${ }^{2}$

TCM usually mimics acute myocardial infarction (MI) at clinical presentation. The most common presenting symptom of TCM is acute chest pain, that usually follows a stressful condition (emotional or physical), electrocardiography (ECG) changes (ST-segment elevation or T-wave inversion or both), and elevation of the cardiac markers thus mimicking MI. ${ }^{3}$ Patients may also present with dyspnea, pulmonary edema and, more rarely, cardiogenic shock..$^{4,5}$

We describe a case of TCM in a young female who developed the condition after acute emotional stress of intrauterine fetal death.

\section{CASE REPORT}

Cardiology consultation was asked by the department of obstetrics and gynecology on October 2013 for a 28-year-old 32 week pregnant woman because the patient developed sudden chest pain and hypotension when she was told about intrauterine fetal demise. On examination, patient had retrosternal chest pain. Her blood pressure was $80 / 60 \mathrm{mmHg}$ and had a heart rate of 80 beats per minutes. Her past medical

\footnotetext{
* Corresponding author

Dr. Laxman Dubey

Department of Cardiology

College of Medical Sciences and Teaching Hospital

Bharatpur, Chitwan, Nepal

E-mail:dubeylax@yahoo.com
} 
history was not significant. No jugular venous distention or lower extremity edema was noted, and the lungs were clear on auscultation. On cardiac auscultation, there was no murmur, rubs, or gallops. Initial ECG showed sinus rhythm with STsegment elevation in leads II, III, aVF and V3-V6 (Figure 1).

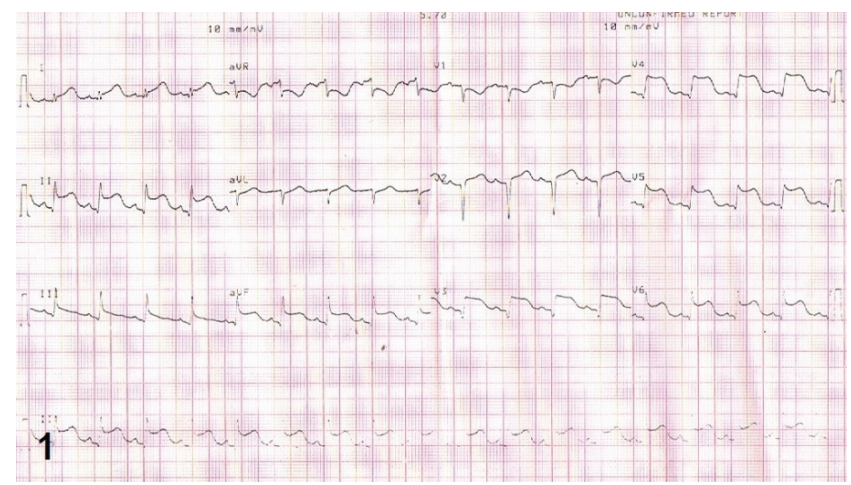

Figure 1. The initial electrocardiography showed sinus rhythm and ST-segment elevations in the leads II, III, aVF, and V3-V6.

The patient's complete blood count, basic metabolic panel and liver function tests were all within the normal range. However, CK-MB was $87 \mathrm{U} / \mathrm{L}$ and troponin I was positive.

Transthoracic echocardiography showed akinetic apex but hyperkinetic mid and basal segments. Since the patient had chest pain, ST-segment elevation in the ECG and cardiac biomarkers were positive, patient was transferred to the catheterization laboratory for coronary angiography (CAG). Cardiac catheterization revealed normal coronaries with Thrombolysis in Myocardial Infarction grade III flow in all coronary arteries (Figure 2).

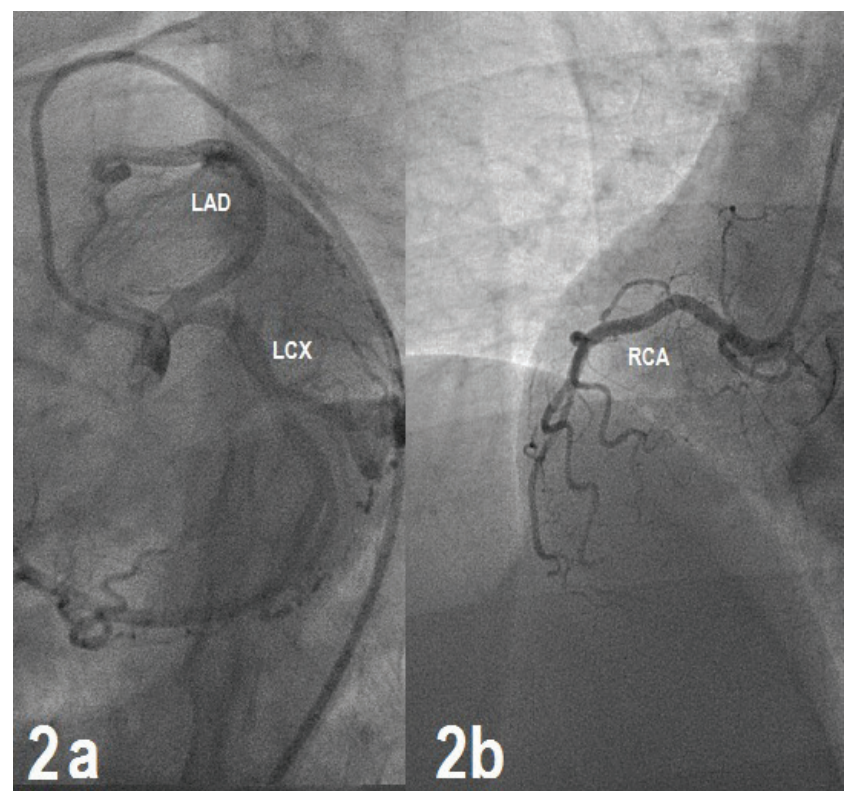

Figure 2a. Coronary angiography revealing normal coronary arteries with TIMI III flow in the left anterior descending (LAD), left circumflex (LCX) coronary arteries and $\mathbf{2 b}$. normal nondominant right coronary artery (RCA)
Right coronary artery was non dominant. Left ventriculography showed end-systolic akinetic apical territory but hyperkinetic remaining wall (Figure 3 ).

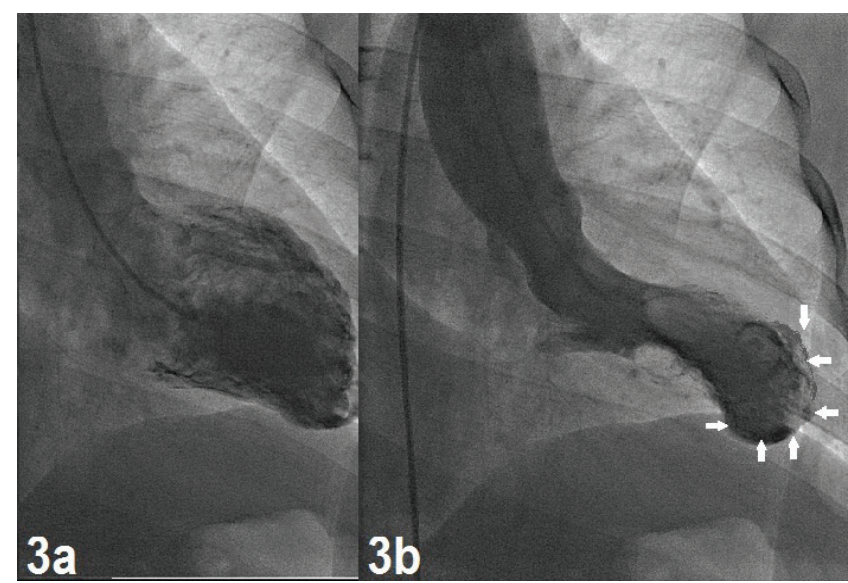

Figure 3a. Left ventriculography in diastole and $\mathbf{3 b}$. in systole showed akinetic apical and inferior segments of the left ventricle

Based on the ECG changes, raised cardiac biomarkers, akinetic apex on echocardiography and left ventriculography with normal coronary arteries and preceding emotional stress, the diagnosis of TCM was made and managed conservatively and her subsequent stay in the hospital was uneventful. Follow-up echocardiography after one week revealed normalization of left ventricular wall motion.

\section{DISCUSSION}

TCM is a rare form of reversible cardiomyopathy that has been primarily described in Japan and is usually characterized by transient LV apical ballooning. ${ }^{6-8}$ Since Tsuchihashi et al reported their largest case series with 88 patients, awareness and clinical interest in this unique syndrome have been raised globally. ${ }^{8}$ Clinical manifestation of TCM is characterized by several criteria ${ }^{9}: 1$. reversible balloon-like LV apical wall motion abnormality with hypercontraction of the basal segments; 2 . chest pain and ST-T segment abnormalities on ECG mimicking acute $\mathrm{Ml} ; 3$. absence of obstructive coronary artery disease or angiographic evidence of acute plaque rupture; 4 . limited cardiac enzyme release relative to the extent of wall motion abnormalities; 5 . an induction by physical or emotional stress in most cases; and 6. having a favorable prognosis.

Although there is no consensus as to the mechanism responsible for transient ballooning, catecholamine toxicity provoked by emotional or physical stress has been proposed as the most likely cause of myocardial stunning in this syndrome., ${ }^{4,10,11}$ Catecholamine toxicity leading to multivessel coronary vasospasm and abnormalities in coronary microvascular function had been proposed as explanations. ${ }^{4,10,11}$ Higher admission levels of plasma catecholamine in patients with 
TCM compared to patients with Killip class III MI had been reported ${ }^{4,11}$ which support that exaggeration of sympathetic stimulation is central to the cause of this syndrome. Moreover, the high concentration of catecholamines in the myocardium would lead to intracellular calcium overload which, in turn, causes a reduction of myocardial contractility. ${ }^{12}$ It is also not very clearly understood why the apex of the LV is so impaired while the remainder is relatively spared. Adrenoceptor density varies throughout the heart with greater density in the apex, possibly explaining the vulnerability of this territory to the sympathetic surge. ${ }^{13}$

The literature describes only a few cases of TCM in younger patients. ${ }^{5}$ It has been reported that TCM occurs primarily in postmenopausal women in the seventh and eighth decades of life and is often associated with an acute physiological or emotional stress ${ }^{14}$, however, in this report we described a case of TCM in a young woman at the age of 28 years, with typical clinical presentation and course. ECG showed STsegment elevation in leads II, III, aVF, V3-V6, and transthoracic echocardiography revealed akinesia of the apex. Common presentation is chest pain typical of acute coronary syndrome. In most cases, presentation is preceded by an emotionally or physically stressful condition such as significant arguments, death of a loved one, natural disasters, legal problems, accidents, surgical procedures, a stay in a critical care unit, and use of illicit drugs. ${ }^{14}$ Our patient developed sudden chest pain after knowing intrauterine fetal death.

\section{REFERENCES}

1. Dote $K$, Sato $H$, Tateishi $H$, et al. Myocardial stunning due to simultaneous multivessel coronary spasm: a review of 5 cases. J Cardiol 1991;2:203-14.

2. Ibanez B, Benezet-Mazuecos J, Navarro F, et al. Takotsubo syndrome: a Bayesian approach to interpreting its pathogenesis. Mayo Clin Proc 2006;81:732-5.

http://dx.doi.org/10.4065/81.6.732

3. Kurisu S, Sato H, Kawagoe T, et al. Tako-tsubo-like left ventricular dysfunction with ST-segment elevation: a novel cardiac syndrome mimicking acute myocardial infarction. Am Heart J 2002; 143:448-55.

http://dx.doi.org/10.1067/mhj.2002.120403

4. Gianni M, Dentali F, Grandi AM, et al. Apical ballooning syndrome or takotsubo cardiomyopathy: a systematic review. Eur Heart J 2006;27:1523-9.

http://dx.doi.org/10.1093/eurheartj/ehl032
No consensus exists regarding appropriate therapy for TCM because the number of reported cases is low and the disorder is possibly underdiagnosed. ${ }^{15}$ Resolution of stressor(s) and conservative therapy usually results in clinical, electrocardiograph, and echocardiograph improvements. The current preferred therapeutic approach is to empirically treat cardiomyopathy with LV systolic dysfunction including $\beta$-blockers and ACE-inhibitors. Diuretics can be added if there is volume overload. However, Fazio et $\mathrm{al}^{16}$ found that treatment with any of these medications does not make a significant difference in outcome. Intravenous inotropes might be required if a patient presents with hypotension.

\section{CONCLUSION}

TCM is a rare but potentially fatal condition, initially very difficult to distinguish it from acute MI. This case study documents a presentation of TCM in a young woman who developed this condition after knowing intrauterine fetal demise. Her clinical presentation of electrocardiographic ST-segment elevation, typical apical LV wall akinesia with resolution, absence of obstructive coronary artery disease on CAG, and preceding emotional stress meet the diagnostic criteria for TCM. Because of close similarity, TCM is confused with acute ST-segment elevation $\mathrm{MI}$ and many patients are thrombolyzed unnecessarily. Therefore, TCM should be kept in mind in the differential diagnosis of acute $\mathrm{MI}$ in patients with chest pain and a recent emotional stress.

5. Bybee KA, Prasad A. Stress-related cardiomyopathy syndromes. Circulation 2008;118:397-409.

http://dx.doi.org/10.1161/CIRCULATIONAHA.106.677625

6. Abe $\mathrm{Y}, \mathrm{Kondo} \mathrm{M}$, Matsuoka R, et al. Assessment of clinical features in transient left ventricular apical ballooning. J Am Coll Cardiol 2003;41:737-42.

http://dx.doi.org/10.1016/S0735-1097(02)02925-X

7. Akashi YJ, Nakazawa K, Sakakibara M, et al. The clinical features of takotsubo cardiomyopathy. QJM 2003;96:563-73.

http://dx.doi.org/10.1093/qjmed/hcg096

8. Tsuchihashi K, Ueshima K, Uchida T, et al. Transient left ventricular apical ballooning without coronary artery stenosis: a novel heart syndrome mimicking acute myocardial infarction. Angina pectoris-myocardial infarction investigations in Japan. J Am Coll Cardiol 2001;38:11-8.

http://dx.doi.org/10.1016/S0735-1097(01)01316-X 
9. Obunai K, Schweitzer P. A case of takotsubo cardiomyopathy. Cardiol 2006;15:31-3.

10. Abe $\mathrm{Y}$, Kondo M. Apical ballooning of the left ventricle: a distinct entity? Heart 2003; 89:974-6.

http://dx.doi.org/10.1136/heart.89.9.974

11. Wittstein IS, Thiemann DR, Lima JA, et al. Neurohumoral features of myocardial stunning due to sudden emotional stress. N Engl J Med 2005;352:539-48.

http://dx.doi.org/10.1056/NEJMoa043046

12. Timek T, Vahl CF, Bonz A, et al. Triiodothyronine reverses depressed contractile performance after excessive catecholamine stimulation. Ann Thorac Surg 1998;66:1618-25.

http://dx.doi.org/10.1016/S0003-4975(98)00764-4

13. Mori H, Ishikawa S, Kojima S, et al. Increased responsiveness of left ventricular apical myocardium to adrenergic stimuli. Cardiovasc Res 1993;27:192-8.

http://dx.doi.org/10.1093/cvr/27.2.192

14. Merchant EE, Johnson SW, Nguyen P, et al. Takotsubo cardiomyopathy: a case series and review of the ziterature. West J Emerg Med 2008;9:104-11.

15. Metzl MD, Altman EJ, Spevack DM, et al. A case of Takotsubo cardiomyopathy mimicking an acute coronary syndrome. Nat Clin Pract Cardiovasc Med 2006;3:53-6. http://dx.doi.org/10.1038/ncpcardio0414

16. Fazio G, Pizzuto C, Barbaro G, et al. Chronic pharmacological treatment in Takotsubo cardiomyopathy. Int J Cardiol 2008;127:121-3.

http://dx.doi.org/10.1016/j.ijcard.2007.04.013 